\title{
Alterazioni neurovascolari nell'epatite cronica C: uno studio caso-controllo
}

\section{Neurovascular alterations in chronic hepatitis C: a case-control study}

\section{Valentina Vedovetto ${ }^{a}$, Franca Bilora ${ }^{a, *}$, Liliana Chemello ${ }^{b}$, Luisa Cavalletto ${ }^{b}$, Alberto Rossato $^{a}$, Francesco Veronese ${ }^{a}$, Anna Gottardo ${ }^{b}$, Antonio Pagnan ${ }^{a}$}

\footnotetext{
${ }^{a}$ Clinica Medica II, Dipartimento di Scienze Cardiologiche, Toraciche, Vascolari, Università degli Studi di Padova

${ }^{\mathrm{b}}$ Clinica Medica V, Dipartimento di Medicina Clinica e Sperimentale, Università degli Studi di Padova
}

\section{KEYWORDS \\ Chronic hepatitis C; Intima-media-thickness; \\ Flow-mediated \\ dilatation test; \\ Autonomic nervous \\ system; \\ Cardiovascular risk; \\ Endothelial dysfunction.}

\begin{abstract}
Summary
Introduction: Hepatitis C is a major health problem: approximately 170 million people are infected with the hepatitis $C$ virus worldwide. It is unclear whether chronic hepatitis $C$ affects atherosclerosis and whether it can cause endothelial and/or autonomic nervous system (ANS) dysfunction.

Materials and methods: From April 2008 through April 2009, we studied 76 patients with biopsyconfirmed chronic hepatitis $C$ and no evidence of cirrhosis, ascites, portal hypertension, encephalopathy, or hepatocellular carcinoma. The age-, sex-, BMI- and cardiovascular risk factor-matched control group comprised 76 healthy, HCV-negative individuals with no evidence of liver, autoimmune, or immunoproliferative diseases and no history of cardiovascular events. Twenty five of the hepatitis $C$ patients were treatment-naive; the other 51 had been treated with interferon (but only 25 had persistent virological responses). Color Doppler sonography was used to measure the intima-media-thickness (IMT) of the common and internal carotid arteries. Endothelial function was assessed in the brachial artery with the flow-mediated-dilatation (FMD) test. The ANS was assessed with the tilt, laying to standing, Valsalva, hand grip, deep breath, and stroop tests. Results: The case group (mean age $52 \pm 13$ years) had a significantly higher internal carotid IMT $(0.86 \pm 0.3$ vs $0.67 \pm 0.1 \mathrm{~mm}$ for controls; $p=0.002)$. Chronic hepatitis $C$ was also associated with an odds ratio for carotid plaque formation (reflected by an IMT $\geq 1.3 \mathrm{~mm}$ ) of 2.15 . Cases also had significantly reduced FMD in the brachial artery $(0.46 \pm 0.9$ vs $0.76 \pm 0.7$ for controls; $p=0.005)$ and significantly altered sympathetic and parasympathetic function ( $p=0.001$ vs controls in the Valsalva, hand grip, deep breath, and stroop tests). Within the case group, all alterations were more severe in patients with significant viremia.
\end{abstract}

\footnotetext{
* Corrispondenza: Clinica Medica II, via Giustiniani 2 - 35128 Padova.

E-mail: franca.bilora@sanita.padova.it (F. Bilora).
} 
Discussion: Our findings suggest that chronic hepatitis $\mathrm{C}$ may be a nonclassic cardiovascular risk factor since it seems to influence the onset of pre-atherosclerotic lesions and to promote atherosclerotic plaque formation in patients with pre-existing increases in carotid IMT. It also seems to cause dysfunctions of the vascular endothelium and ANS.

Conclusions: Chronic hepatitis C may increase cardiovascular risk and promote ANS dysfunctions, particularly when patients have experienced treatment failure and have persistent viremia. These patients may require cardiovascular and neurologic follow-up.

(c) 2010 Elsevier Srl. All rights reserved.

\section{Introduzione}

Il virus dell'epatite $C$ costituisce una delle più frequenti cause di epatite cronica, con più di 170 milioni di persone affette nel mondo [1]. La terapia con interferone non sempre risulta efficace; la risposta alla terapia dipende infatti da alcune variabili, tra cui: il genotipo virale, con maggiori possibilità di guarigione nei genotipi 2 e 3 rispetto a 1 e 4; l'età; lo stadio e il grado dell'epatite; alcune caratteristiche del paziente non ancora chiarite. La preparazione di un vaccino non è ancora possibile a causa della grande capacità di mutazione del virus [1,2]. Pertanto l'epatite causata da HCV è, attualmente, uno dei più importanti problemi di salute mondiale [3] e per tale ragione il presente studio affronta specificamente l'epatopatia indotta da questo virus.

Recentemente la letteratura suggerisce un rapporto fra aterosclerosi e numerosi agenti infettivi (compresi quelli che determinano altre tipologie di epatite cronica, diverse da quella da HCV), sulla base del processo infiammatorio che essi comportano a livello sistemico: è nota, infatti, la fisiopatologia infiammatoria della placca aterosclerotica $[4,5]$. Tuttavia, il rapporto tra HCV e patogenesi dell'aterosclerosi non è ancora chiaro e spesso è oggetto di controversie [6]: secondo alcuni studi l'HCV risulterebbe avere un ruolo fondamentale nella patogenesi dell'aterosclerosi [7-11]; secondo altri l'HCV non dimostrerebbe alcuna correlazione con il processo ateromasico [12-17], a differenza di altri virus epatopatici, o potrebbe addirittura risultare un fattore protettivo in virtù dell'ipocolesterolemia indotta dallo stato avanzato dell'epatopatia (cirrosi epatica) $[18,19]$.

È ormai nota la disfunzione endoteliale precoce in corso di aterosclerosi $[5,20,21]$ ed è risaputo che la storia naturale della cirrosi comporta una disfunzione del sistema nervoso [22], con alterazione del tono vasomotore e della heart rate variability e con aumento del rischio di morte precoce $[23,24]$; poco si sa, invece, delle possibili alterazioni precoci in corso di epatopatia cronica precedente lo stadio di cirrosi.

Scopo di questo studio caso-controllo è analizzare la relazione tra epatopatia cronica da virus HCV e aterosclerosi effettuando le seguenti valutazioni: valutazione, mediante eco-color-Doppler, dello spessore intima-media (IntimaMedia Thickness, IMT) delle carotidi come test capace di fornire informazioni aggiuntive sul rischio cardiovascolare (pre-danno) di un soggetto rispetto ai classici fattori di rischio; valutazione della funzione endoteliale, stabilita mediante Flow Mediated Dilatation (FMD), che determina la capacità vasodilatatrice dell'arteria brachiale poiché, se ridotta, è in grado di predire patologie cardiovascolari [25]; valutazione del sistema nervoso autonomo, che in molti studi correla con la funzione endoteliale [26-29].

\section{Materiali e metodi}

Abbiamo esaminato in un anno (aprile 2008-aprile 2009) 76 pazienti affetti da epatite cronica C, selezionati dall'Ambulatorio delle epatiti virali HCV della Clinica Medica V e dal Reparto di Malattie Infettive dell'Azienda Ospedaliera di Padova. Per la diagnosi di HCV sono stati utilizzati test sierologici e virologici (ELISA, RIBA, RT-PCR). I pazienti sono stati appaiati con 76 controlli provenienti da un gruppo di 500 pazienti non HCV positivi, non epatopatici, della Clinica Medica II dell'Azienda Ospedaliera di Padova, in base a sesso, età, fattori di rischio cardiovascolari quali fumo, ipertensione, diabete mellito, dislipidemia, indice di massa corporea $(\mathrm{BMI})$ (si è cercato di appaiare, secondo categorie omogenee, il grado di tabagismo attuale o pregresso non oltre i 10 anni precedenti, il grado di pressione arteriosa, di glicemia media, di colesterolemia totale e/o HDL), in modo tale che fosse possibile eliminare dall'indagine possibili fattori confondenti. Per definire i fattori di rischio cardiovascolare, abbiamo fatto riferimento ai criteri indicati in letteratura [5,20,21]. Criteri di inclusione dei casi sono stati: età compresa tra 25 e 80 anni; storia di epatite cronica C senza evoluzione allo stadio di cirrosi epatica e/o ulteriori complicanze (ascite, ipertensione portale, encefalopatia, tumore epatico ecc.) e senza coinfezioni virali da HBV o HIV; assenza di malattie autoimmuni, crioglobulinemie, vasculiti o disordini linfoproliferativi o stato di gravidanza; assenza di storia pregressa o attuale di assunzione di alcol e/o sostanze stupefacenti. I controlli non HCV positivi non epatopatici, a loro volta, non dovevano presentare una storia di malattie cardiovascolari (come infarto del miocardio, ictus, angina, ischemia transitoria, aneurisma dell'aorta) né patologie di carattere autoimmune o linfoproliferativo.

Per quanto riguarda il trattamento, 51 casi di epatite cronica C erano stati trattati con interferone, mentre 25 pazienti non avevano mai ricevuto alcun tipo di trattamento; tra i pazienti trattati, 25 avevano raggiunto la persistente eliminazione del virus nel tempo e 26 non avevano risposto alla terapia, poiché mostravano valori di viremia non ridotti di più di 2 logaritmi entro il terzo mese di trattamento, come da linea guida [30].

Sono stati esaminati i valori delle proteine sieriche, dell'albuminemia, della bilirubinemia, delle transaminasi AST e ALT, del tempo di protrombina, della colesterolemia e della glicemia in tutti i pazienti, e i pazienti epatopatici sono stati sottoposti a ecografia epatica e a FibroScan per la corretta stadiazione dell'epatopatia in atto.

L'esame dei tronchi sovraortici è stato effettuato in ogni paziente mediante scansioni ecografiche in sezioni assiali e longitudinali e calcolando l'IMT con apparecchio Esaote 
Technos (Giappone), con sonda a $8,5 \mathrm{MHz}$ per ecografia B-mode e sonda a $6 \mathrm{MHz}$ per il doppler pulsato, mantenendo un angolo di incidenza della sonda di circa $60^{\circ}$. I pazienti sono stati fatti accomodare in posizione supina, con il collo ruotato di $45^{\circ}$ controlateralmente all'arteria in esame, mentre l'operatore era seduto alle loro spalle. Lo studio è stato condotto in ambiente tranquillo, con temperatura media di $22-25^{\circ} \mathrm{C}$. È stata dapprima effettuata una valutazione bidimensionale di entrambe le arterie carotidi comuni, interne ed esterne (laddove esplorabili), secondo scansioni assiali e longitudinali (queste ultime secondo i piani anteriore, laterale e posteriore). L'IMT della parete vasale è stato definito come la distanza dell'interfaccia tra lume-intima e media-avventizia a livello del far wall (parete del vaso distante dalla sonda o posteriore), che permette di registrare un'immagine più nitida, mentre il diametro del vaso è definito come la distanza tra le pareti luminali. È stato quindi misurato l'IMT della parete posteriore dell'arteria carotide comune nel suo tratto distale, situato circa $2 \mathrm{~cm}$ prossimalmente alla biforcazione. Sono state effettuate 3 misurazioni adiacenti dell'IMT, a livello di un tratto di parete esteso per $2 \mathrm{~cm}$ e libero da placche, ed è stato, quindi, ottenuto il valore medio. Seguendo i criteri della letteratura scientifica, abbiamo considerato lesioni preaterosclerotiche (semplice ispessimento) quelle con valori di IMT $\geq 1,0 \mathrm{~mm}$ e $<1,3 \mathrm{~mm}$, e lesioni aterosclerotiche o placche vere e proprie quelle con valori di IMT $\geq 1,3 \mathrm{~mm}$ [31].

L'arteria brachiale del braccio dominante è stata successivamente esaminata vicino alla fossa antecubitale, con il braccio in posizione comoda, mediante scansioni longitudinali. Poiché numerosi fattori condizionano la reattività vascolare, per valutare la funzione endoteliale (FMD), in accordo con le linee guida dell'American College of Cardiology [25], i pazienti dovevano digiunare da 8 a 12 ore prima dell'esame e sospendere l'assunzione di medicamenti vasoattivi (qualora possibile), caffeina, cibi grassi, vitamina C e tabacco nelle 4-6 ore precedenti; dovevano inoltre evitare l'esercizio fisico. L'esame deve essere condotto in ambiente tranquillo, a temperatura controllata. Il diametro sistolico dell'arteria brachiale è stato misurato prima e dopo lo stimolo ischemico. È stato dimostrato che, all'aumentare dell'IMT delle carotidi (e quindi all'aumentare del rischio di placca), diminuisce la FMD, ovvero la capacità di dilatazione dell'arteria brachiale in risposta all'iperemia successiva allo stimolo ischemico e indotta da un rilascio di ossido di azoto (NO) $[25,32]$. Pertanto, dopo misurazione basale del diametro dell'arteria brachiale, è stato applicato uno stimolo ischemico mediante insufflazione di un bracciale da sfigmomanometro a livello dell'avambraccio, fino a raggiungere una pressione di $50 \mathrm{mmHg}$ sopra la sistolica, per circa 3 minuti, durante i quali il paziente veniva monitorato. Sgonfiando successivamente il bracciale, è stata indotta iperemia reattiva, con aumento dello stress parietale e vasodilatazione NO-mediata. Il diametro arterioso postiperemia è stato misurato tra 45 e 60 secondi dopo lo sgonfiamento del bracciale. La vasodilatazione flusso-mediata è stata espressa come differenza dei due diametri (FMD = diametro postiperemia - diametro preiperemia).

Per la valutazione del sistema nervoso autonomo, i pazienti non dovevano assumere farmaci simpatomimetici e parasimpatomimetici, antistaminici, medicine per la tosse, steroidi, diuretici nelle 24 ore precedenti i test. Inoltre

Tabella 1 Test autonomici.

\begin{tabular}{|c|c|c|}
\hline Test & Segmento autonomico esplorato & Descrizione \\
\hline Tilt & $\begin{array}{l}\text { Funzioni simpatica } \\
\text { e parasimpatica, PA } \\
\text { e variazioni della FC }\end{array}$ & $\begin{array}{l}\text { Il paziente resta disteso sul lettino per il Tilt test per } 30 \text { minuti: } \\
\text { questa condizione induce un sequestro di sangue agli arti inferiori. } \\
\text { Il test è normale se la PA non scende più di } 20 / 10 \mathrm{mmHg}\end{array}$ \\
\hline Laying to Standing & $\begin{array}{l}\text { Funzioni simpatica } \\
\text { e parasimpatica, PA } \\
\text { e variazioni della FC }\end{array}$ & $\begin{array}{l}\text { Un deficit di vasocostrizione nel passaggio da clinostatismo a } \\
\text { ortostatismo causa un eccessivo calo della PA ( }>30 / 15 \mathrm{mmHg})\end{array}$ \\
\hline Valsalva & Sistema parasimpatico & $\begin{array}{l}\text { Il paziente respira a glottide chiusa, mantenendo una pressione } \\
\text { di } 40 \text { mmHg per } 15 \text { secondi. Il test è normale se: } \\
\text { - in fase } 1 \text { la PA aumenta; } \\
\text { - in fase } 2 \text { la Pa decresce verso un plateau ed è presente tachicardia; } \\
\text { - in fase } 3 \text { la PA decresce; } \\
\text { - in fase } 4 \text { si hanno aumento della PA e bradicardia }\end{array}$ \\
\hline Hand Grip & Sistema simpatico & $\begin{array}{l}\text { Il test consiste in un esercizio isometrico. Il paziente deve stringere } \\
\text { in modo intermittente una piccola palla di gomma. La pressione } \\
\text { diastolica dovrebbe aumentare }(15 \mathrm{mmHg})\end{array}$ \\
\hline Deep Breath & Sistema parasimpatico & $\begin{array}{l}\text { Il test consiste nell'esame della fisiologica aritmia respiratoria. } \\
\text { Il paziente dovrebbe respirare profondamente } 6 \text { volte/min. } \\
\text { Differenze }<10 \text { battiti/min tra l'inspirio e l'espirio sono } \\
\text { considerate anormali }\end{array}$ \\
\hline Stroop & Sistema simpatico & $\begin{array}{l}\text { Si tratta di un esercizio di calcolo mentale. La PA dovrebbe } \\
\text { aumentare mentre il paziente legge e pronuncia il nome di } \\
\text { colori che sono scritti su fogli aventi colori non corrispondenti } \\
\text { al nome stesso }\end{array}$ \\
\hline
\end{tabular}


dovevano evitare l'assunzione di caffè, tè, alcol, tabacco nelle 3 ore precedenti gli esami. La registrazione dei parametri basali (elettrocardiogramma, pressione arteriosa, frequenza cardiaca) è stata effettuata in continuo mentre i pazienti erano supini sul lettino per Tilt test. I test autonomici effettuati sono descritti in dettaglio nella tabella 1 $[33,34]$. I valori sono espressi come rapporti basati sugli intervalli R-R dell'elettrocardiogramma.

Gli autori dichiarano che lo studio presentato è stato realizzato in accordo con gli standard etici stabiliti nella Dichiarazione di Helsinki, e che il consenso informato è stato ottenuto da tutti i partecipanti prima del loro arruolamento allo studio.

\section{Statistica}

I dati di questo studio sono espressi come media \pm deviazione standard o come percentuali, mentre per i test statistici è stata utilizzata la mediana, misura di tendenza centrale meno sensibile ai valori estremi. L'analisi statistica è stata effettuata applicando: il test $\mathrm{T}$ di Student per le variabili indipendenti; l'odds ratio; il test del Chi quadrato. Il livello di significatività è stato posto per $p<0,05$. L'analisi statistica è stata condotta con il supporto del software R Development Core Team Program [35].

\section{Risultati}

Sono stati esaminati, nel periodo intercorrente tra il $1^{\circ}$ aprile 2008 e il 30 aprile 2009, 76 pazienti affetti da epatite cronica $\mathrm{C}$ afferenti ai nostri Ambulatori (diagnosi pregressa con test sierologici, virologici e istologici), con età media di $52 \pm 13$ anni (minimo 27, massimo 81), di cui 45 maschi e 31 femmine. Di questi pazienti, 25 avevano precedentemente eseguito la terapia per l'HCV e sono stati definiti guariti in quanto hanno definitivamente negativizzato l'HCV-RNA del siero per più di 6 mesi e hanno mostrato la persistente normalizzazione delle ALT; 26 pazienti erano stati invece trattati senza efficacia terapeutica (persistenza del virus nel siero); infine, 25 pazienti non avevano ricevuto alcuna terapia specifica.

I casi di epatite cronica $C$ sono stati appaiati il più accuratamente possibile con altrettanti controlli (HCV-RNA negativi non epatopatici) per età, sesso, fattori di rischio cardiovascolare e $\mathrm{BMI}$, in modo da eliminare tali dati confondenti. Nella tabella 2 sono riportate le caratteristiche dei pazienti.

\section{Relazione tra epatite cronica C e aterosclerosi}

Nell'esame dell'IMT i pazienti con epatite cronica $C$ hanno mostrato valori di spessore intimale maggiori rispetto ai controlli, soprattutto a livello delle carotidi comuni e di quelle interne (tabella 3 ). Inoltre, tra i casi trattati è stata osservata una differenza significativa $(p=0,01)$ tra i pazienti guariti e i pazienti che non hanno negativizzato il virus (questi ultimi presentano valori di aterosclerosi maggiori). Infine, se si procede all'analisi dei risultati suddividendo i valori di IMT in due categorie (ispessimento semplice: valori di IMT > 1,0 e < 1,3 mm; placche: valori di IMT $\geq 1,3 \mathrm{~mm}$ ), i pazienti con epatite cronica $C$ hanno dimostrato un rischio maggiore di sviluppo di placche carotidee rispetto ai controlli (odds ratio 2,15: positivo per aumento del rischio). L'analisi dei genotipi, per quanto importanti nel determinare il successo o il fallimento della terapia, come già specificato nell'introduzione $[1,36,37]$, non ha dimostrato differenze significative nello studio dell'IMT.

\section{Relazione tra epatite cronica $\mathrm{C}$ e disfunzione endoteliale}

Dall'analisi della FMD, tra pazienti con epatite cronica C e controlli non HCV positivi, è emersa una differenza statisticamente significativa (tabella 3): in effetti, i primi hanno dimostrato una minore capacità di dilatazione dopo stimolo ischemico rispetto ai controlli. Inoltre, esiste una correlazione significativa tra valori di FMD più bassi e valori di IMT più elevati $(p=0,03)$.

Tabella 2 Caratteristiche dei pazienti.

\begin{tabular}{lcc}
\hline & Casi con epatite cronica $C^{*}$ & Controlli non epatopatici (non HCV positivi) \\
\hline M/F $(\mathrm{N})$ & $45 / 31$ & $45 / 31$ \\
\hline Età (anni) & $52,0 \pm 13,0$ & $51,71 \pm 13,5$ \\
\hline BMI $\left(\mathrm{kg} / \mathrm{m}^{2}\right)$ & $24,6 \pm 3,6$ & $24,4 \pm 3,6$ \\
\hline Fumatori o ex fumatori $(\mathrm{N})$ & $33(24 \mathrm{M}, 9 \mathrm{~F})$ & $33(24 \mathrm{M}, 9 \mathrm{~F})$ \\
\hline Ipertesi $(\mathrm{N})$ & $26(16 \mathrm{~F}, 10 \mathrm{M})$ & $26(16 \mathrm{~F}, 10 \mathrm{M})$ \\
\hline Dislipidemici $(\mathrm{N})$ & $11(7 \mathrm{~F}, 4 \mathrm{M})$ & $11(7 \mathrm{~F}, 4 \mathrm{M})$ \\
\hline Diabetici $(\mathrm{N})$ & $5(3 \mathrm{M}, 2 \mathrm{~F})$ & $5(3 \mathrm{M}, 2 \mathrm{~F})$ \\
\hline Pressione sistolica $(\mathrm{mmHg})$ & $130,0 \pm 16,4$ & $124,2 \pm 10,9$ \\
\hline Pressione diastolica $(\mathrm{mmHg})$ & $77,4 \pm 12,0$ & $75,5 \pm 5,5$ \\
\hline Frequenza cardiaca $(\mathrm{bpm})$ & $68,0 \pm 9,8$ & $71,2 \pm 6,2$ \\
\hline
\end{tabular}

Legenda: $M=$ maschi; $F=$ femmine.

Dati espressi come numero di pazienti $(\mathrm{N})$ o media \pm deviazione standard. 
Tabella 3 Valutazioni vascolari: esame dell'IMT nella carotide comune e interna (valori medi bilaterali) e test FMD nei casi e nei controlli (test: T di Student).

\begin{tabular}{lccc}
\hline Valutazione & Casi con epatite cronica C & Controlli non epatopatici (non HCV positivi) $^{*}$ & P \\
\hline IMT CC & $0,86 \pm 0,2($ IC $0,8-0,90)$ & $0,7 \pm 0,1($ IC $0,68-0,72)$ & 0,04 \\
\hline IMT ICA & $0,86 \pm 0,3($ IC $0,8-0,92)$ & $0,67 \pm 0,1($ IC $0,65-0,69)$ & 0,002 \\
\hline FMD $(\mathrm{mm})$ & $0,46 \pm 0,9($ IC $0,2-0,66)$ & $0,76 \pm 0,7($ IC $0,6-0,92)$ & 0,0005 \\
\hline
\end{tabular}

Legenda: $C C=$ carotide comune; ICA = carotide interna; IC = intervallo di confidenza; FMD = differenza fra la dilatazione precedente lo stimolo ischemico e la dilatazione successiva.

Dati espressi come media \pm deviazione standard.

\section{Relazione tra epatite cronica C e sistema nervoso autonomo}

I test autonomici, in particolar modo i test Deep Breath, Valsalva, Hand Grip e Stroop, hanno dimostrato significative differenze tra soggetti con epatite cronica $C$ e controlli non $\mathrm{HCV}$ positivi (tabella 4), mentre il Tilt test e il Laying to Standing non sono risultati statisticamente significativi.

\section{Discussione}

Oggigiorno l'epatite cronica da virus C rappresenta, senza dubbio, uno dei grandi problemi di salute pubblica a livello mondiale, per il quale non sono ancora note misure preventive efficaci. Inoltre, è ormai riconosciuta l'importanza delle patologie cardiovascolari come prima causa di morte nei Paesi industrializzati. La nostra ipotesi di lavoro consisteva nel valutare l'epatite cronica C come ulteriore fattore di rischio, oltre ai classici fattori di rischio cardiovascolare (fumo, ipertensione, diabete, dislipidemia, BMI).

Dai dati rilevati è emerso che la patologia cronica da virus HCV determina un maggiore ispessimento delle carotidi, rispetto ai controlli non epatopatici non HCV positivi, e ciò potrebbe incidere sullo sviluppo e sull'evoluzione della malattia aterosclerotica aggiungendosi ai consueti fattori di rischio. In particolare, il rischio aumenta nei pazienti che non eliminano il virus in modo definitivo. È possibile che i diversi metodi di studio adottati e le differenti casistiche selezionate (per esempio, stadio dell'epatopatia nei pazienti: epatite cronica o cirrosi) possano motivare i risultati spesso discordanti presenti in letteratura $[6,13]$. Infine, non abbiamo considerato il fattore tempo, ovvero la relazione esistente tra epatite cronica $\mathrm{C}$ e aterosclerosi al tempo zero di diagnosi dell'epatopatia, prima che venga intrapresa qualsiasi misura di tipo igienico-sanitario, fattore cui riserveremo un'attenzione particolare in ulteriori studi già in atto.

I pazienti con epatite cronica C presentano una disfunzione endoteliale significativa: infatti non sono in grado di vasodilatare, dopo stimolo ischemico, allo stesso modo dei controlli. Poiché bassi valori di FMD correlano con alti valori di IMT, come già menzionato, anche il nostro studio conferma la relazione tra disfunzione endoteliale e aterosclerosi presente in letteratura $[25,32]$. Probabilmente, i pazienti con persistenza del virus dopo il trattamento antivirale potrebbero presentare una disfunzione endoteliale maggiore rispetto ai casi rimanenti, anche se nel nostro studio la differenza tra pazienti guariti e non guariti non è stata statisticamente significativa. Ciò potrebbe supportare l'ipotesi, sebbene questa necessiti di ulteriori conferme e analisi specifiche, che la persistenza del virus, nonostante la terapia, possa essere un fattore determinante nelle alterazioni vascolari. Inoltre, il nostro gruppo ha avuto a disposizione pazienti con epatite cronica $C$, ma si potrebbe ipotizzare, tuttavia, che altre epatopatie infettive croniche dello stesso tenore clinico possano determinare i medesimi risultati; ciò non rientrava però nello scopo dello studio.

Infine, come già ipotizza parte della letteratura scientifica più recente $[38,39]$, il nostro studio conferma ampiamente la presenza di alterazioni del sistema nervoso autonomo nei pazienti con epatopatia cronica $C$ e si può affermare, anche in questo caso, che la persistenza del virus determini le alterazioni più evidenti. Il virus probabilmente agisce negativamente sia sul sistema nervoso sia sulla funzione vascolare.

Anche se l'interferone è considerato un importante agente terapeutico, capace di modulare l'infiammazione [40], nota causa nella patogenesi dell'aterosclerosi come già menzionato, non è stato possibile analizzare con precisione il ruolo della terapia nelle alterazioni in esame, data l'esiguità dei dati al riguardo, anche se i soggetti con

Tabella 4 Test autonomici risultati significativi nei casi e nei controlli: i valori si basano sui rapporti degli intervalli R-R dell'elettrocardiogramma (test: T di Student).

\begin{tabular}{lccc}
\hline Test autonomici & Casi di epatite cronica C & Controlli non epatopatici (non HCV positivi) $^{*}$ & P \\
\hline Valsalva & $1,5 \pm 0,2$ (IC 1,46-1,54) & $1,8 \pm 0,12$ (IC 1,77-1,83) & 0,001 \\
\hline Hand Grip & $2,3 \pm 7,3($ IC $0,66-3,94)$ & $11,82 \pm 7,7$ (IC 10,09-13,55) & 0,001 \\
\hline Deep Breath & $1,26 \pm 0,12($ IC $1,23-1,29)$ & $1,42 \pm 0,07($ IC $1,40-1,43)$ & 0,001 \\
\hline Stroop & $1,2 \pm 5,8$ (IC - $0,1-2,5)$ & $6,6 \pm 3,3($ IC $5,86-7,34)$ & 0,001 \\
\hline
\end{tabular}

* Dati espressi come media \pm deviazione standard. 
persistenza di viremia (non efficacia della terapia per le caratteristiche descritte nell'introduzione) hanno mostrato valori di IMT significativamente maggiori. La terapia antivirale è senz'altro importante nel controllo della fibrosi epatica e chiave di volta per il raggiungimento dell'eradicazione virale $[41,42]$, ma si richiede un incremento della casistica (già in atto) per poter studiare il suo ruolo anche nelle alterazioni cardiovascolari e neurologiche dei pazienti con epatite cronica $C$, per verificare quale ruolo possa rivestire al riguardo.

\section{Conclusioni}

Il virus dell'epatite cronica C costituisce un probabile fattore di rischio che si aggiunge a quelli già noti nel determinare alterazioni cardiovascolari, in virtù del quadro infiammatorio cronico associato. Inoltre i pazienti con epatite cronica $\mathrm{C}$ sembrano dimostrare una maggiore disfunzione endoteliale e del sistema nervoso autonomo, rispetto ai controlli non HCV positivi, e l'ispessimento intimale è più evidente soprattutto in caso di persistenza del virus per fallimento della terapia (resistenza). I dati ottenuti inducono a formulare l'ipotesi che sia proprio la persistenza del virus, nonostante la terapia, a giocare un ruolo importante nel determinare le alterazioni da noi studiate, oggetto senz'altro di ulteriori necessari approfondimenti. Si potrebbe pertanto consigliare, alla luce di questa ipotesi, un monitoraggio cardiovascolare e neurologico continuo in tali pazienti, per escludere la progressione a disfunzioni più severe.

\section{Ringraziamenti}

Si ringrazia la dottoressa Veronica Vedovetto per il contributo fondamentale all'analisi statistica.

\section{Conflitto di interessi}

Gli autori dichiarano di essere esenti da conflitto di interessi.

\section{Bibliografia}

1. Czepiel J, Biesiada G, Mach T. Viral hepatitis C. Pol Arch Med Wewn 2008;118(12):734-40.

2. Szabó E, Lotz G, Páska C, Kiss A, Schaff Z. Viral hepatitis: new data on hepatitis C infection. Pathol Oncol Res 2003;9(4): 215-21.

3. Boonstra A, Woltman AM, Janssen HL. Immunology of hepatitis B and hepatitis $C$ virus infections. Best Pract Res Clin Gastroenterol 2008;22(6):1049-61.

4. Boddi M, Abbate R, Chellini B, Giusti B, Solazzo V, Soft F, et al. $\mathrm{HCV}$ infection facilitates asymptomatic carotid atherosclerosis: preliminary report of HCV RNA localization in human carotid plaques. Dig Liver Dis 2007;39(Suppl 1):S55-60.

5. Cotran RS, Kumar V, Collins T. Vasi sanguigni. In: Dall'Olio F, Brigotti $M$, Cevenini R, Farabegoli F, Pession A, Trerè $D$, et al (a cura di). Robbins. Le basi patologiche delle malattie. VI Ed. Padova: Piccin Nuova Libreria, 2000. p. 577-97.

6. Aslam F, Alam M, Lakkis NM. Hepatitis C and carotid atherosclerosis. A retrospective analysis. Atherosclerosis 2010;209(2): $340-3$.
7. Sawayama Y, Okada K, Maeda S, Ohnishi H, Furusyo N, Hayashi J. Both hepatitis $C$ virus and Chlamydia pneumoniae infection are related to the progression of carotid atherosclerosis in patients undergoing lipid lowering therapy. Fukuoka Igaku Zasshi 2006;97(8):245-55.

8. Ishizaka N, Ishizaka Y, Takahashi E, Tooda E, Hashimoto H, Nagai $\mathrm{R}$, et al. Association between hepatitis $\mathrm{C}$ virus seropositivity, carotid-artery plaque, and intima-media thickening. Lancet 2002;359(9301):133-5.

9. Ishizaka Y, Ishizaka N, Takahashi E, Unuma T, Tooda E, Hashimoto $\mathrm{H}$, et al. Association between hepatitis $\mathrm{C}$ virus core protein and carotid atherosclerosis. Circ J 2003;67(1):26-30.

10. Targher G, Bertolini L, Padovani R, Rodella S, Arcaro G, Day C. Differences and similarities in early atherosclerosis between patients with non-alcoholic steatohepatitis and chronic hepatitis B and C. J Hepatol 2007;46(6):1126-32.

11. Alyan O, Kacmaz F, Ozdemir O, Deveci B, Astan R, Celebi AS, et al. Hepatitis $C$ infection is associated with increased coronary artery atherosclerosis defined by modified Reardon severity score system. Circ J 2008;72(12):1960-5.

12. Volzke H, Schwahn C, Wolff B, Mentel R, Robinson DM, Kleine V, et al. Hepatitis $B$ and $C$ virus infection and the risk of atherosclerosis in a general population. Atherosclerosis 2004;174(1): 99-103.

13. Moritani M, Adachi K, Arima N, Takashima T, Miyaoka Y, Niigaki M, et al. A study of arteriosclerosis in healthy subjects with HBV and HCV infection. J Gastroenterol 2005;40(11):1049-53.

14. Momiyama $\mathrm{Y}$, Ohmori $\mathrm{R}$, Kato $\mathrm{R}$, Taniguchi $\mathrm{H}$, Nakamura $\mathrm{H}$, Ohsuzu F. Lack of any association between persistent hepatitis $\mathrm{B}$ or $\mathrm{C}$ virus infection and coronary artery disease. Atherosclerosis 2005;181(1):211-3.

15. Irshad M, Dhar I, Gupta S, Khushboo, Joshi YK. Correlation of serum HCV core concentration with blood level of lipid and antioxidants in various forms of liver diseases. Hepatogastroenterology 2007;54(75):898-902.

16. Arcari CM, Nelson KE, Netski DM, Nieto FJ, Gaydos CA. No association between hepatitis $C$ virus seropositivity and acute myocardial infarction. Clin Infect Dis 2006;43(6):e53-6.

17. Kiechl S, Egger G, Mayr M, Wiedermann CJ, Bonora E, Oberhollenzer F, et al. Chronic infections and the risk of carotid atherosclerosis: prospective results from a large population study. Circulation 2001;103(8):1064-70.

18. Bilora F, Rinaldi R, Boccioletti V, Petrobelli F, Girolami A. Chronic viral hepatitis: a prospective factor against atherosclerosis. A study with echo-color Doppler of the carotid and femoral arteries and the abdominal aorta. Gastroenterol Clin Biol 2002;26(11):1001-4.

19. Bilora F, Campagnolo E, Rinaldi R, Rossato A, Arzenton M, Petrobelli F. Carotid and femoral atherosclerosis in chronic hepatitis C: a 5-year follow-up. Angiology 2008;59(6):717-20.

20. Kumar P, Clark M. Malattie cardiovascolari. In: Medicina clinica. I edizione italiana della VI edizione inglese. Roma: CIC Edizioni Internazionali; 2007. p. 798-803.

21. Woolf N. Il sistema cardiovascolare. In: Anatomia patologica. IX Ed. Napoli: Edises; 2005. p. 327-46.

22. Dümcke CW, Møller S. Autonomic dysfunction in cirrhosis and portal hypertension. Scand J Clin Lab Invest 2008;68(6):437-47.

23. Hendrickse MT, Thuluvath PJ, Triger DR. Natural history of autonomic neuropathy in chronic liver disease. Lancet 1992; 339(8807):1462-4.

24. Hendrickse MT, Triger DR. Peripheral and cardiovascular autonomic impairment in chronic liver disease: prevalence and relation to hepatic function. J Hepatol 1992;16(1-2): 177-83.

25. Corretti MC, Anderson TJ, Benjamin EJ, Celermajer D, Charbonneau F, Creager MA, et al., International Brachial Artery Reactivity Task Force. Guidelines for the ultrasound assessment of endothelial-dependent flow-mediated vasodilation of the 
brachial artery: a report of the International Brachial Artery Reactivity Task Force. J Am Coll Cardiol 2002;39(2):257-65.

26. Ko SH, Song KH, Park SA, Kim SR, Cha BY, Son HY, et al. Cardiovascular autonomic dysfunction predicts acute ischaemic stroke in patients with Type 2 diabetes mellitus: a 7-year follow-up study. Diabet Med 2008;25(10):1171-7.

27. Lin YD, Hsu KL, Wu ET, Tsai MS, Wang CH, Chang CY, et al. Autonomic neuropathy precedes cardiovascular dysfunction in rats with diabetes. Eur J Clin Invest 2008;38(9):607-14.

28. Suvorova IA, Govorin AV, Zatsev DN. Vegetative disturbances and dysfunction of the endothelium in patients with postinfarction cardiosclerosis. Kardiologiia 2008;48(10):10-2.

29. Ohira T, Diez Roux AV, Prineas RJ, Kizilbash MA, Carnethon MR, Folsom AR. Associations of psychosocial factors with heart rate and its short-term variability: multi-ethnic study of atherosclerosis. Psychosom Med 2008;70(2):141-6.

30. Italian Association for the Study of the Liver, Italian Society of Infectious, Tropical Diseases, Italian Society for the Study of Sexually Transmitted Diseases. Practice guidelines for the treatment of hepatitis C: recommendations from an AISF/ SIMIT/SIMAST Expert Opinion Meeting. Dig Liver Dis 2010; 42(2):81-91.

31. Yang KC, Chen MF, Su TC, Jeng JS, Hwang BS, Lin LY, et al. Hepatitis B virus seropositivity is not associated with increased risk of carotid atherosclerosis in Taiwanese. Atherosclerosis 2007;195(2):392-7.

32. Hashimoto M, Eto M, Akishita M, Kozaki K, Ako J, lijima K, et al. Correlation between flow-mediated vasodilatation of the brachial artery and intima-media thickness in the carotid artery in men. Arterioscler Thromb Vasc Biol 1999;19(11):2795-800.
33. Victor M, Ropper AH. Adams e Victor. Principi di neurologia. Il manuale. VII Ed. Milano: McGraw-Hill; 2002. p. 217-28.

34. Bergamini L, Bergamasco B, Mutani R. Fisiopatologia del sistema nervoso autonomo. In: Manuale di neurologia clinica. III Ed. Torino: Libreria Cortina; 2001. p. 153-71.

35. R Development Core Team. R: A language and environment for statistical computing. Wien: R Foundation for Statistical Computing, 2009. http://www.R-project.org

36. Fargion S, Fracanzani AL, Valenti L. Treatment choices for people infected with HCV. J Antimicrob Chemother 2004;53(5):708-12.

37. Ferenci P. Peginterferon and ribavirin in HCV: improvement of sustained viral response. Best Pract Res Clin Gastroenterol 2008;22(6):1109-22.

38. Frith J, Newton JL. Autonomic dysfunction in chronic liver disease. Liver Int 2009;29(4):483-9.

39. Oliver MI, Miralles R, Rubies-Prat J, Navarro X, Espadaler JM, Sola $\mathrm{R}$, et al. Autonomic dysfunction in patients with non-alcoholic chronic liver disease. J Hepatol 1997;26(6):1242-8.

40. Zhang LN, Velichko S, Vincelette J, Fitch RM, Vergona R, Sullivan $M E$, et al. Interferon-beta attenuates angiotensin II-accelerated atherosclerosis and vascular remodeling in apolipoprotein $\mathrm{E}$ deficient mice. Atherosclerosis 2008;197(1):204-11.

41. Maylin S, Martinot-Peignoux M, Moucari R, Boyer N, Ripault MP, Cazals-Hatem D, et al. Eradication of hepatitis $C$ virus in patients successfully treated for chronic hepatitis C. Gastroenterology 2008;135(3):821-9.

42. Guido M, De Franceschi L, Olivari N, Leandro G, Felder M, Corrocher R, et al. Effects of interferon plus ribavirin treatment on NF-kappaB, TGF-beta1, and metalloproteinase activity in chronic hepatitis C. Mod Pathol 2006;19(8):1047-54. 